Open Access

\title{
Pre-existing atrial fibrillation and risk of arterial thromboembolism and death in intensive care unit patients: a population-based cohort study
}

\author{
Jacob Gamst ${ }^{1,2,3,4^{*}}$ (D), Christian Fynbo Christiansen ${ }^{1}$, Bodil Steen Rasmussen ${ }^{3}$, Lars Hvilsted Rasmussen ${ }^{4,5}$
} and Reimar Wernich Thomsen ${ }^{1}$

\begin{abstract}
Introduction: Pre-existing atrial fibrillation (AF) may worsen prognosis in patients admitted to the intensive care unit (ICU).

Methods: In a cohort study (2005-2011) including all patients with first-time ICU admissions in Denmark $(n=57,110)$, we compared patients with and without pre-existing AF and estimated absolute risks and relative risks (RRs) of arterial thromboembolism and death within 30 days and 365 days following admission, using Kaplan-Meier methods and multivariate regression analyses. We analysed the prognostic impact of AF within strata of patient age, sex, coexisting cardiac diseases, and ICU therapies.

Results: Among ICU patients, 5065 (9\%) had pre-existing AF. Compared with patients without AF, those with AF were older (median age 75 vs. 62 years) and had more comorbidity. The risk of arterial thromboembolism was $2.8 \%$ in patients with AF and $2.0 \%$ in non-AF patients at 30 days, and $4.3 \%$ and $2.9 \%$, respectively, at 365 days. Corresponding RRs were 1.41 crude and 1.14 (95\% confidence interval [CI] 0.93-1.40) adjusted at 30 days, and 1.50 crude and 1.20 (95 \% Cl 1.02-1.41) adjusted at 365 days. Thirty-day mortality was $27 \%$ in patients with pre-existing AF and $16 \%$ in non-AF patients (crude RR 1.67, adjusted RR 1.04, $95 \% \mathrm{Cl} 0.99-1.10)$. Corresponding mortality estimates at 365 days were $40.9 \%$ and $25.4 \%$, respectively (crude RR 1.61, adjusted RR 1.03, $95 \% \mathrm{Cl}$ 1.00-1.07). In stratified analyses, pre-existing AF increased mortality in ICU patients aged $<55$ years (adjusted RR at 30 days $1.73,95 \% \mathrm{Cl} 1.29-2.32$; adjusted RR at 365 days $1.34,95 \% \mathrm{Cl} 1.06-1.69$ ) and in ICU patients treated with mechanical ventilation (adjusted RR at 30 days $1.12,95 \% \mathrm{Cl} 1.05-1.20$, adjusted RR at 365 days 1.09, $95 \%$ Cl: 1.04-1.15). Analyses stratified by sex and coexisting cardiac diseases yielded adjusted RRs close to 1.
\end{abstract}

Conclusions: In ICU patients, pre-existing AF was associated with modestly increased risk of arterial thromboembolism when adjusted for the substantially higher age and comorbidity levels in patients with AF, whereas there was no overall association with mortality. In ICU patients aged $<55$ years and in those treated with mechanical ventilation, AF predicted increased mortality.

\footnotetext{
* Correspondence: jacob.gamst@rn.dk

${ }^{1}$ Department of Clinical Epidemiology, Institute of Clinical Medicine, Aarhus

University Hospital, Olof Palmes Allé 43-45, DK-8200 Aarhus N, Denmark

${ }^{2}$ Department of Cardiology, Aalborg University Hospital, Hobrovej 18-22,

DK-9000 Aalborg, Denmark

Full list of author information is available at the end of the article
}

(c) 2015 Gamst et al. Open Access This article is distributed under the terms of the Creative Commons Attribution 4.0 International License (http://creativecommons.org/licenses/by/4.0/), which permits unrestricted use, distribution, and reproduction in any medium, provided you give appropriate credit to the original author(s) and the source, provide a link to the Creative Commons license, and indicate if changes were made. The Creative Commons Public Domain Dedication waiver (http://creativecommons.org/publicdomain/zero/1.0/) applies to the data made available in this article, unless otherwise stated. 


\section{Introduction}

The overall burden of pre-existing chronic diseases is an important determinant of prognosis for patients admitted to the intensive care unit (ICU) $[1,2]$. Chronic diseases are reportedly more prevalent in ICU patients than in the general population, and with the ageing of the population, the prevalence of chronic diseases is expected to increase in coming years $[1,3]$. Despite this, the prognostic influence of individual coexisting diseases in ICU patients remains poorly investigated.

Atrial fibrillation (AF) is the most common heart rhythm disorder, affecting $1.0-1.8 \%$ of the adult population in western countries $[4,5]$. In the general population, AF has been associated with substantially increased risk of mortality, stroke, and heart failure [6-8]. In patients admitted to the ICU, new-onset AF has been linked to increased risk of death $[9,10]$. To date, evidence on how, and to what extent, pre-existing AF affects the prognosis for ICU patients is sparse. Pre-existing AF could potentially have serious consequences in critically ill patients. The ability to compensate for haemodynamic alterations during critical illness may be reduced in patients with $\mathrm{AF}$, and the inflammatory response could increase the risk of thrombosis formation during ICU stay [11]. These possible complications of AF are likely more pronounced with increased severity of illness during the ICU admission. Also, on the one hand, as the prognostic impact of AF increases with advancing age, it is possible that the effect of AF is influenced by patient age $[12,13]$. On the other hand, the potentially detrimental effects of AF may be modified by effective medications prescribed for AF, such as anticoagulants and heart rhythm-modulating drugs.

The prevalence of AF is expected to increase considerably in the coming years with the increasing ageing population and prevalence of comorbidities, and elderly patients with comorbidities are increasingly admitted to the ICU $[5,14,15]$. It is therefore of major importance to elucidate the prognostic impact of AF in ICU patients of different ages and in those with and without comorbidities. Thus, we conducted a large, population-based cohort study to examine the effect of pre-existing AF and associated pharmacotherapy on the risk of arterial thromboembolism (ATE) and death following ICU admission. We also sought to investigate whether any prognostic effect of AF differed according to patient age, sex, and comorbidity and with regard to the reason for and treatment during ICU admission.

\section{Methods}

This nationwide cohort study was conducted in Denmark, a nation with approximately 5.6 million inhabitants. The study was based on prospectively collected data from medical and administrative databases. Each Danish resident is assigned a unique personal identifying number, which enables unambiguous crosslinking of public registries for research purposes. The Danish national health care system offers unrestricted, tax-funded hospital and primary care to all its citizens.

\section{Identification of the cohort and ICU data}

All adult patients (age 15+ years) with a first-time ICU admission in Denmark between January 2005 and December 2011 were included in the cohort. We used the Danish Intensive Care Database (DID) to identify the cohort members. The DID maintains data on all ICU admissions in Denmark from 2005 onward [16]. From the DID, we also retrieved data on treatment with mechanical ventilation, non-invasive ventilation, inotropes and/or vasopressors, and renal replacement therapy during the index ICU admission. To differentiate between ICU admissions related to recent surgery and medical ICU admissions, we obtained data from the Danish National Patient Register (DNPR) on surgical procedures performed within 7 days preceding the ICU admission. The DNPR includes all patients admitted to Danish hospitals since 1977 and holds dates of admission and discharge, surgical procedure codes, and diagnostic codes [17]. We assessed surgical procedures as any type of surgery and specified according to cardiac, abdominal, orthopaedic, or vascular surgery.

\section{Data on atrial fibrillation}

We used the DNPR to identify cohort members with previously diagnosed AF. We considered diagnoses of AF assigned during either in-hospital admission or at a hospital specialist outpatient clinic visit within 5 years before the index ICU admission. Because AF and atrial flutter share the same code in the DNPR, we were not able to distinguish between these arrhythmias. However, it has been demonstrated that, among patients coded with AF or atrial flutter in the DNPR, 92-95\% have AF $[18,19]$. Data on pre-admission treatment for AF was acquired from the Danish Health Service Prescription Registry, which holds data on all prescriptions dispensed from all Danish pharmacies [20]. We noted prescriptions for the most frequently used medications for AF (vitamin $\mathrm{K}$ antagonists, aspirin, beta-blockers, nondihydropyridine calcium-channel blockers, amiodarone, and digoxin) [21]. We also included data on statins, which are both frequently prescribed to individuals with cardiovascular disease and associated with favourable prognosis in ICU patients [22].

\section{Data on potential confounders}

To control our estimates for confounding by coexisting diseases, we retrieved data from the DNPR on diagnoses from previous hospital contacts (in-hospital admission, 
outpatient clinic, or emergency department) at any time before ICU admission. For the thromboembolism analyses, we identified the risk factors included in the $\mathrm{CHA}_{2} \mathrm{DS}_{2}$-VASc score (i.e., congestive heart failure, hypertension, age, diabetes, prior stroke/transient ischaemic attack/thromboembolism, vascular disease, and sex category), which has been validated for prediction of stroke in patients with $\operatorname{AF}[13,23]$. For the mortality analyses, we included data on the 19 disease categories in the Charlson comorbidity index, which have been validated for prediction of hospital and ICU mortality [24-26]. For the mortality analyses, we further included diagnoses of valvular heart disease, angina pectoris and chronic ischaemic heart disease, hypertension, alcoholism, and obesity. To address any imbalances in frailty or health awareness not accounted for by previously diagnosed diseases, we additionally retrieved data from the National Health Service Register, which includes consultations and procedures provided by primary health care providers [27], on preventive consultations, social medicine-related consultations, application for reimbursement due to chronic or terminal illness, conversational therapy, and influenza vaccinations by the general practitioner (GP) within 1 year preceding the index ICU admission.

\section{Outcomes}

The outcomes were ATE and death. We evaluated both outcomes at 30 days and 365 days following the ICU admission date. We defined ATE as a diagnosis of non-haemorrhagic stroke or thrombosis or embolism in arteries of the extremities, the mesenteric arteries, or unspecified arteries recorded in the DNPR. The Civil Registration System provided data on vital status of the cohort members. This database, which is updated daily, encompasses all Danish residents since 1968 and holds information on exact dates of birth, death, and migration [28].

\section{Statistical analyses}

Follow-up began at ICU admission and continued until 1 January 2013. We computed the cumulative risk of ATE in patients with and without pre-existing AF, accounting for the competing risk of death in the analyses [29]. To further compare the risk of ATE in patients with $\mathrm{AF}$ with that in non-AF patients, we calculated cumulative risk ratios (CRRs), both crude and adjusted for prevalence of the risk factors in the $\mathrm{CHA}_{2} \mathrm{DS}_{2}$-VASc score. Both cumulative risks and CRRs were computed by analysing pseudo values for each observation in a generalized linear model $[30,31]$. Within each of the cohorts of patients with and without pre-existing AF, we further investigated the effect of pre-admission use of vitamin $\mathrm{K}$ antagonists and aspirin on the risk of ATE by comparing users with non-users. Because the DNPR records all diagnoses assigned during an admission to the same date, some episodes of ATE may have been present before the patient was transferred to the ICU if the ATE and ICU admission occurred during the same hospital admission. In sensitivity analyses, we therefore estimated the cumulative risk and CRR of ATE at 30 days and 365 days following ICU admission, considering only ATE diagnosed at a later hospital admission than the hospital admission during which the patient was treated at the ICU.

Using the Kaplan-Meier method, we estimated mortality risks in patients with pre-existing $\mathrm{AF}$ and non-AF patients and compared these by computing relative risks (RRs) through generalized linear regression analyses of pseudo observations [30, 32]. The RRs were presented as crude estimates and estimates adjusted for age, sex, comorbidities (the 19 disease categories in the Charlson comorbidity index, valvular heart disease, angina pectoris and chronic ischaemic heart disease, hypertension, alcoholism, and obesity), and frailty markers as defined by GP services (preventive consultations, social medicinerelated consultations, reimbursement due to chronic or terminal illness, conversational therapy, and influenza vaccinations). To investigate whether any effect of $A F$ was different across subgroups of ICU patients, we divided the cohort into three age groups of equal size $(15-54,55-70$, and 71-103 years) and repeated the analyses within each age group. Further, we conducted the analyses within strata defined by sex, previously diagnosed myocardial infarction and congestive heart failure, use of mechanical ventilation, non-invasive ventilation, inotropes and vasopressor agents, and renal replacement therapy during the ICU admission, as well as type of surgery performed in relation to the ICU admission. To examine to effect of pre-admission treatment for $\mathrm{AF}$, we assessed absolute risks and RRs of death in users of statins, vitamin $\mathrm{K}$ antagonists, aspirin, beta-blockers, non-dihydropyridine calciumchannel blockers, amiodarone, and digoxin as compared with non-users in patients with and without AF separately.

Statistical analyses were performed using Stata 11.2 software (StataCorp, College Station, TX, USA), including the user-written st0202 package.

\section{Ethics}

The study was approved by the Danish Data Protection Agency (record number 2009-41-3987). Informed consent was not required for this registry study according to Danish legislation.

\section{Results}

The study included 57,110 ICU patients, of whom 5065 (9 \%) had pre-existing AF (Table 1). Follow-up was 
Table 1 Characteristics of 57,110 ICU patients with and without pre-existing atrial fibrillation

\begin{tabular}{|c|c|c|c|}
\hline & $\mathrm{AF}, \mathrm{n}(\%)$ & No $A F, n(\%)$ & Total, n (\%) \\
\hline Participants & $5065(8.9)$ & $52,045(91.1)$ & $57,110(100.0)$ \\
\hline Male & $3162(62.4)$ & $28,851(55.4)$ & $32,013(56.1)$ \\
\hline Age, yr, median (IQR) & $75(67-81)$ & $62(47-73)$ & $64(49-75)$ \\
\hline \multicolumn{4}{|l|}{ Age groups } \\
\hline $15-55 \mathrm{yr}$ & $257(5.1)$ & $18,247(35.1)$ & $18,504(32.4)$ \\
\hline $55-71 \mathrm{yr}$ & $1534(30.3)$ & $17,212(33.1)$ & $18,746(32.8)$ \\
\hline $71-103$ yr & $3274(64.6)$ & $16,586(31.9)$ & $19,860(34.8)$ \\
\hline \multicolumn{4}{|l|}{ Comorbid conditions } \\
\hline Prior myocardial infarction & $1056(20.1)$ & $5082(9.8)$ & $6138(10.8)$ \\
\hline Congestive heart failure & $1714(33.8)$ & $3213(6.2)$ & $4927(8.6)$ \\
\hline Peripheral artery disease & $824(16.3)$ & $4874(9.4)$ & $5698(10.0)$ \\
\hline Valvular heart disease & $1215(24.0)$ & $3756(7.2)$ & $4971(8.7)$ \\
\hline Hypertension & $2457(48.5)$ & $10,997(21.1)$ & $13,454(23.6)$ \\
\hline Angina pectoris & $1506(29.7)$ & $7690(14.8)$ & $9196(16.1)$ \\
\hline Chronic ischaemic heart disease & $1724(34.0)$ & $6292(12.1)$ & $8016(14.0)$ \\
\hline Cerebrovascular disease & $1217(24.0)$ & $6386(12.3)$ & $7603(13.3)$ \\
\hline Hemiplegia & $35(0.7)$ & $420(0.8)$ & $455(0.8)$ \\
\hline Transient cerebral ischaemic attack & $359(7.1)$ & $1932(3.7)$ & $2291(4.0)$ \\
\hline Dementia & $109(2.2)$ & $542(1.0)$ & $651(1.1)$ \\
\hline Chronic pulmonary disease & $1336(26.4)$ & $7769(14.9)$ & $9105(16.0)$ \\
\hline Connective tissue disease & $360(7.1)$ & $2294(4.4)$ & $2654(4.7)$ \\
\hline Moderate to severe liver disease & $43(0.8)$ & $614(1.2)$ & $657(1.1)$ \\
\hline Mild liver disease & $117(2.3)$ & $1597(3.1)$ & $1714(3.0)$ \\
\hline Diabetes & $830(16.4)$ & $4748(9.1)$ & $5578(9.8)$ \\
\hline Diabetes mellitus types 1 and 2 with end-organ damage & $522(10.3)$ & $2673(5.1)$ & $3195(5.6)$ \\
\hline Moderate to severe renal disease & $518(10.2)$ & $2110(4.1)$ & $2628(4.5)$ \\
\hline Any tumour & $967(19.1)$ & $8750(16.8)$ & $9717(17.0)$ \\
\hline Leukaemia & $27(0.5)$ & $330(0.63)$ & $357(0.6)$ \\
\hline Lymphoma & $90(1.8)$ & $651(1.3)$ & $741(1.3)$ \\
\hline Metastatic solid tumour & $149(2.9)$ & $1604(3.1)$ & $1753(3.1)$ \\
\hline AIDS & $1(0.0)$ & $64(0.1)$ & $65(0.1)$ \\
\hline \multicolumn{4}{|l|}{$\mathrm{CHA}_{2} \mathrm{DS}_{2}$-VASc score } \\
\hline 0 & $226(4.5)$ & $11,608(22.3)$ & $11,834(20.7)$ \\
\hline 1 & $575(11.4)$ & $15,704(30.2)$ & $16,279(28.5)$ \\
\hline $2+$ & $4264(84.1)$ & $24,733(47.5)$ & $28,997(50.8)$ \\
\hline \multicolumn{4}{|l|}{ Lifestyle-related diagnoses } \\
\hline Alcoholism & $221(4.4)$ & $3155(6.1)$ & $3376(5.9)$ \\
\hline Obesity & $491(9.7)$ & $2908(5.6)$ & $3399(6.0)$ \\
\hline \multicolumn{4}{|l|}{ Consultations and services at the $\mathrm{GP}^{\mathrm{a}}$} \\
\hline Seasonal influenza vaccination & $2557(50.1)$ & $13,510(26.0)$ & $16,067(28.1)$ \\
\hline Preventive consultation & $305(6.0)$ & $2894(5.6)$ & $3199(5.6)$ \\
\hline Social medicine-related consultation & $646(12.8)$ & $3773(7.3)$ & $4419(7.7)$ \\
\hline Conversational therapy & $70(1.4)$ & $1646(3.2)$ & $1716(3.0)$ \\
\hline Reimbursement for chronic illness & $73(1.4)$ & $621(1.2)$ & $694(1.2)$ \\
\hline Reimbursement for terminal illness & $9(0.2)$ & $70(0.1)$ & $79(0.1)$ \\
\hline
\end{tabular}


Table 1 Characteristics of 57,110 ICU patients with and without pre-existing atrial fibrillation (Continued)

\begin{tabular}{|c|c|c|c|}
\hline \multicolumn{4}{|l|}{ Pre-admission cardiovascular drug use ${ }^{b}$} \\
\hline Statins & $1716(33.9)$ & $11,031(21.2)$ & $12,747(22.3)$ \\
\hline Aspirin & $1988(39.3)$ & $10,708(20.6)$ & $12,696(22.2)$ \\
\hline Vitamin $\mathrm{K}$ antagonists & $2500(49.4)$ & $1339(2.6)$ & $3839(6.7)$ \\
\hline Beta-blockers & $2742(54.1)$ & $9254(17.8)$ & $11,996(21.0)$ \\
\hline Non-dihydropyridine calcium-channel blockers & $360(7.1)$ & $901(1.7)$ & $1261(2.2)$ \\
\hline Digoxin & $1668(33.0)$ & $779(1.5)$ & $2447(4.3)$ \\
\hline Amiodarone & $363(7.2)$ & $115(0.2)$ & $478(0.8)$ \\
\hline \multicolumn{4}{|l|}{ ICU therapy } \\
\hline Mechanical ventilation & $2114(41.7)$ & $18,410(35.4)$ & $20,524(36.0)$ \\
\hline Non-invasive ventilation & $550(11.0)$ & $3772(7.3)$ & $4322(7.6)$ \\
\hline Renal replacement therapy & $254(5.0)$ & $1432(2.8)$ & $1686(3.0)$ \\
\hline Treatment with inotropes or vasopressors & $2057(40.6)$ & $14,987(28.8)$ & $17,044(29.8)$ \\
\hline \multicolumn{4}{|l|}{ Surgery performed 0-7 days before ICU admission } \\
\hline Any surgical procedure & $3039(60.0)$ & $32,160(61.8)$ & $35,199(61.6)$ \\
\hline Cardiac surgery & $1038(20.5)$ & $6669(12.8)$ & 7707 (13.5) \\
\hline Vascular surgery & $179(3.5)$ & $2764(5.3)$ & $2943(5.2)$ \\
\hline Abdominal surgery & $899(17.8)$ & $9608(18.5)$ & $10,507(18.4)$ \\
\hline Orthopaedic surgery & $237(4,7)$ & $2594(5.0)$ & $2831(5.0)$ \\
\hline Non-surgical ICU admission & $2026(40.0)$ & $19,885(38.2)$ & $21,911(38.4)$ \\
\hline
\end{tabular}

$A F$ atrial fibrillation, $I Q R$ interquartile range, GP general practitioner

${ }^{a}$ Within 1 year preceding ICU admission

${ }^{\mathrm{b}}$ Defined as reimbursed prescription within 100 days preceding the ICU admission

incomplete for 172 individuals (169 emigrated and 3 disappeared). The participants' characteristics are presented in Table 1.

\section{Risk of arterial thromboembolism}

At 30 days following ICU admission, the cumulative risk of ATE was $2.8 \%$ (95 \% CI 2.4-3.3\%) in patients with pre-existing $\mathrm{AF}$ and $2.0 \%$ (95 \% CI $1.9-2.2 \%)$ in patients without AF (Table 2). The corresponding CRR adjusted for prevalence of the risk factors included in the $\mathrm{CHA}_{2} \mathrm{DS}_{2}$-VASc score was 1.14 (95\% CI 0.93-1.40). Risks of ATE at 365 days were 4.3 \% (95 \% CI 3.7-4.4\%) and $2.9 \%$ (95 \% CI 2.7-3.0\%) for patients with and without pre-existing AF, respectively, with a confounderadjusted CRR of 1.20 (95\% CI 1.02-1.41). Considering only ATE diagnosed at a later hospital admission than the hospital admission during which the patient was treated in the ICU, the 30-day risk of ATE was $0.3 \%$ (95\% CI 0.1-0.5\%) in patients with pre-existing AF and $0.2 \%$ (95\% CI 0.2-0.3\%) in patients without AF, with an adjusted CRR of 0.94 (95\% CI 0.35-2.52), and 365-day risks were $1.7 \%$ (95\% CI 1.3-2.0\%) and $1.0 \%$ (95 \% CI 0.9-1.1\%) for patients with and without pre-existing $\mathrm{AF}$, respectively, with an adjusted CRR of 1.24 (95 \% CI 0.921.67) (Additional file 1: Table S1).
In patients with $\mathrm{AF}$ who were pre-admission users of aspirin, the adjusted CRR of ATE was 1.05 (95\% CI $0.64-1.75)$ at 30 days and 1.20 (95 \% CI 1.02$1.41)$ at 365 days following ICU admission compared with patients with AF who were non-users of aspirin. Among AF patients, pre-admission use of vitamin $\mathrm{K}$ antagonists was associated with adjusted CRRs of 0.57 (95 \% CI $0.26-1.25)$ at 30 days and 0.76 (95 \% CI $0.50-1.16)$ at 365 days following admission.

\section{Mortality}

The 30-day mortality was $26.8 \%$ (95 \% CI $25.6 \%-28.0 \%$ ) in patients with pre-existing $\mathrm{AF}$ and $16.0 \%$ (95\% CI $15.7 \%-16.4 \%$ ) in patients without AF (Table 3). The confounder-adjusted RR for 30-day mortality of patients with and without pre-existing AF was 1.04 (95 \% CI $0.99-1.10$ ). In the youngest third of the cohort (ages 15-55 years), increased mortality was observed with pre-existing AF (adjusted RR 1.73, 95 \% CI 1.29-2.32), whereas pre-existing AF did not increase mortality in the two older age groups (55-71 years and 71-105 years) after controlling for confounders (adjusted RR 1.09, 95 \% CI 0.96-1.24; adjusted RR 1.04, $95 \%$ CI 0.98-1.10). In those who received mechanical ventilation during the ICU admission, AF was associated with increased 30-day 
Table 2 Risk of arterial thromboembolism at 30 days and 365 days following ICU admission in patients with and without atrial fibrillation and by pre-admission use of aspirin and vitamin $\mathrm{K}$ antagonists

\begin{tabular}{|c|c|c|c|c|}
\hline & \multicolumn{2}{|l|}{ 30-day ATE risk } & \multicolumn{2}{|l|}{ 365-day ATE risk } \\
\hline & $\mathrm{AF}$ & No AF & AF & No AF \\
\hline \multicolumn{5}{|l|}{ Overall } \\
\hline Cumulative risk, \% & $2.8(2.4-3.3)$ & $2.0(1.9-2.2)$ & $4.3(3.7-4.9)$ & $2.9(2.7-3.0)$ \\
\hline \multicolumn{5}{|l|}{ Cumulative risk ratio } \\
\hline Crude & $1.41(1.19-1.67)$ & 1 (ref) & $1.50(1.31-1.73)$ & 1 (ref) \\
\hline Adjusted $^{a}$ & $1.14(0.93-1.40)$ & 1 (ref) & $1.20(1.02-1.41)$ & 1 (ref) \\
\hline \multicolumn{5}{|l|}{ Aspirin users } \\
\hline \multicolumn{5}{|l|}{ Cumulative risk, \% } \\
\hline Non-users & $2.5(2.0-3.1)$ & $2.0(1.8-2.1)$ & $3.7(3.1-4.4)$ & $2.7(2.5-2.8)$ \\
\hline Users & $3.4(2.6-4.2)$ & $2.4(2.1-2.7)$ & $5.1(4.1-6.1)$ & $3.5(3.2-3.9)$ \\
\hline \multicolumn{5}{|l|}{ Cumulative risk ratio } \\
\hline Non-users & 1 (ref) & 1 (ref) & 1 (ref) & 1 (ref) \\
\hline Users, crude & $1.35(0.98-1.86)$ & $1.21(1.05-1.40)$ & $1.36(1.05-1.77)$ & $1.31(1.17-1.47)$ \\
\hline Users, adjusted ${ }^{a}$ & $1.05(0.64-1.75)$ & $0.78(0.65-0.93)$ & $1.13(0.81-1.59)$ & $0.81(0.70-0.95)$ \\
\hline \multicolumn{5}{|c|}{ Vitamin $\mathrm{K}$ antagonist users } \\
\hline \multicolumn{5}{|l|}{ Cumulative risk, \% } \\
\hline Non-users & $3.4(2.7-4.1)$ & $2.0(1.9-2.2)$ & $4.9(4.1-5.7)$ & $2.9(2.7-3.0)$ \\
\hline Users & $2.4(1.8-3.0)$ & $1.9(1.2-2.7)$ & $3.7(2.9-4.4)$ & $3.1(2.2-4.0)$ \\
\hline \multicolumn{5}{|l|}{ Cumulative risk ratio } \\
\hline Non-users & 1 (ref) & 1 (ref) & 1 (ref) & 1 (ref) \\
\hline Users, crude & $0.70(0.50-0.96)$ & $0.95(0.65-1.39)$ & $0.75(0.58-0.97)$ & $1.07(0.79-1.46)$ \\
\hline Users, adjusted $^{\mathrm{a}}$ & $0.57(0.26-1.25)$ & $0.71(0.46-1.12)$ & $0.76(0.50-1.16)$ & $0.82(0.58-1.16)$ \\
\hline
\end{tabular}

mortality even after confounder adjustment (adjusted RR 1.12, 95 \% CI 1.05-1.20).

At 1 year following ICU admission, the mortality was $40.9 \%$ (95 \% CI 40.0-42.3 \%) in patients with preexisting AF and $25.4 \%$ (95 \% CI 25.0-25.8 \%) in patients without AF (Table 3). The corresponding adjusted RR was 1.03 (95\% CI 1.00-1.07). Similar to the 30-day results, pre-existing AF was associated with a pronounced increase in mortality among individuals aged 15-55 years (adjusted RR 1.34, 95 \% CI 1.06-1.69), whereas this was not the case in the older patients. Among patients receiving mechanical ventilation during the ICU admission, mortality at 1 year following ICU admission was increased in patients with AF compared with non-AF patients (adjusted RR 1.09, $95 \%$ CI 1.04-1.15).

In patients with pre-existing $\mathrm{AF}$, pre-admission users of vitamin $\mathrm{K}$ antagonists had lower 30-day and 1-year mortality compared with non-users (adjusted RR 0.91, 95 \% CI 0.82-1.00; adjusted RR 0.91, 95 \% CI 0.85-0.97) (Table 4). Reduced 30-day mortality in patients with AF was also seen with user of non-dihydropyridine calcium- channel blockers, but the estimate was rather imprecise (adjusted RR 0.86, 95 \% CI 0.71-1.03), and the effect did not persist at 1 year of follow-up. Patients with AF using amiodarone had higher 30-day and 1-year mortality than non-users (adjusted RR 1.15, 95 \% CI 0.98-1.34; adjusted RR 1.12, 95 \% CI 1.01-1.25). Among patients with preexisting AF, use of statins, aspirin, beta-blockers, and digoxin did not seem to influence the mortality at 30 days or 1 year following ICU admission.

\section{Discussion}

This study of 57,110 ICU patients provides novel insights into the role of pre-existing AF on the prognosis for patients admitted to the ICU. Without confounder adjustment, our findings show that patients with preexisting AF are at substantially higher risk of ATE and death following ICU admission than are patients without pre-existing AF. However, much of the excess risk of ATE and most of the excess risk of death is conveyed by AF's being part of a complex that includes advanced age and a high burden of cardiovascular comorbidity. Of 
Table 3 Mortality at 30 days and 365 days following admission in 57,110 adult ICU patients with and without atrial fibrillation

\begin{tabular}{|c|c|c|c|c|}
\hline & $A F, \%$ & No $A F, \%$ & Crude $R^{a}$ & Adjusted RR $R^{a, b}$ \\
\hline \multicolumn{5}{|l|}{ 30-day mortality } \\
\hline Overall & $26.8(25.6-28.0)$ & $16.0(15.7-16.4)$ & $1.67(1.59-1.76)$ & $1.04(0.99-1.10)$ \\
\hline \multicolumn{5}{|l|}{ Age } \\
\hline $15-54 \mathrm{yr}$ & $13.6(10.0-18.5)$ & $6.1(5.7-6.4)$ & $2.25(1.64-3.08)$ & $1.73(1.29-2.32)$ \\
\hline $55-70 \mathrm{yr}$ & $16.7(14.9-18.7)$ & $14.6(14.1-15.1)$ & $1.14(1.02-1.29)$ & $1.09(0.96-1.24)$ \\
\hline $71-105 \mathrm{yr}$ & $32.6(31.0-34.2)$ & $28.5(27.8-29.2)$ & $1.14(1.08-1.21)$ & $1.04(0.98-1.10)$ \\
\hline \multicolumn{5}{|l|}{ Sex } \\
\hline Female & $30.0(28.0-32.1)$ & $16.7(16.2-17.2)$ & $1.80(1.67-1.93)$ & $1.05(0.97-1.14)$ \\
\hline Male & $24.9(23.4-26.4)$ & $15.5(15.1-16.0)$ & $1.60(1.50-1.71)$ & $1.04(0.97-1.12)$ \\
\hline \multicolumn{5}{|c|}{ Prior myocardial infarction } \\
\hline Not present & $26.2(24.9-27.6)$ & $15.6(15.3-16.0)$ & $1.68(1.59-1.77)$ & $1.04(0.98-1.11)$ \\
\hline Present & $29.1(26.4-31.9)$ & $20.0(18.9-21.1)$ & $1.46(1.31-1.63)$ & $1.06(0.93-1.19)$ \\
\hline \multicolumn{5}{|c|}{ Congestive heart failure } \\
\hline Not present & $24.2(22.8-25.7)$ & $15.3(15.0-15.6)$ & $1.58(1.49-1.69)$ & $1.04(0.97-1.10)$ \\
\hline Present & $31.9(29.7-34.1)$ & $27.7(26.2-29.3)$ & $1.15(1.05-1.26)$ & $1.08(0.99-1.18)$ \\
\hline \multicolumn{5}{|c|}{ Mechanical ventilation } \\
\hline No & $24.9(24.3-25.5)$ & $11.2(10.9-11.5)$ & $2.10(1.96-2.26)$ & $1.00(0.93-1.08)$ \\
\hline Yes & $31.3(29.4-33.3)$ & $23.6(22.1-25.1)$ & $1.26(1.18-1.35)$ & $1.12(1.05-1.20)$ \\
\hline \multicolumn{5}{|c|}{ Renal replacement therapy } \\
\hline No & $25.9(24.7-27.1)$ & $15.3(15.0-15.6)$ & $1.69(1.60-1.78)$ & $1.04(0.98-1.09)$ \\
\hline Yes & $44.1(38.2-50.4)$ & $41.7(39.2-44.3)$ & $1.06(0.91-1.23)$ & $1.04(0.88-1.22)$ \\
\hline \multicolumn{5}{|c|}{ Treatment with inotropes or vasopressors } \\
\hline No & $24.3(22.8-25.9)$ & $12.1(11.7-12.4)$ & $2.02(1.88-2.16)$ & $1.02(0.94-1.09)$ \\
\hline Yes & $30.4(28.5-32.4)$ & $25.9(25.2-26.6)$ & $1.17(1.09-1.26)$ & $1.08(1.01-1.16)$ \\
\hline \multicolumn{5}{|c|}{ Non-invasive ventilation } \\
\hline No & $25.2(24.0-26.5)$ & $14.7(14.4-15.0)$ & $1.72(1.63-1.81)$ & $1.06(1.00-1.12)$ \\
\hline Yes & $39.6(35.7-43.9)$ & $33.3(31.8-34.8)$ & $1.19(1.06-1.33)$ & $0.98(0.88-1.10)$ \\
\hline \multicolumn{5}{|c|}{ Surgery performed, any type } \\
\hline No & $36.0(33.9-38.1)$ & $21.2(20.6-21.7)$ & $1.70(1.59-1.81)$ & $0.99(0.93-1.06)$ \\
\hline Yes & $20.7(19.3-22.2)$ & $12.9(12.5-13.3)$ & $1.60(1.49-1.73)$ & $1.03(0.95-1.12)$ \\
\hline \multicolumn{5}{|c|}{ Cardiac surgery performed } \\
\hline No & $31.9(30.5-33.3)$ & $17.5(17.1-17.8)$ & $1.82(1.74-1.92)$ & $1.02(0.97-1.08)$ \\
\hline Yes & $7.1(5.7-8.9)$ & $6.3(5.7-6.9)$ & $1.13(0.89-1.44)$ & $0.80(0.52-1.23)$ \\
\hline \multicolumn{5}{|c|}{ Abdominal surgery performed } \\
\hline No & $26.2(24.9-27.6)$ & $15.8(15.5-16.2)$ & $1.65(1.56-1.75)$ & $1.05(0.99-1.11)$ \\
\hline Yes & $29.6(26.7-32.7)$ & $16.7(16.2-17.7)$ & $1.75(1.56-1.95)$ & $1.02(0.91-1.14)$ \\
\hline \multicolumn{5}{|c|}{ Orthopaedic surgery performed } \\
\hline No & $26.5(25.3-27.7)$ & $16.1(15.7-16.4)$ & $1.65(1.57-1.74)$ & $1.05(1.00-1.11)$ \\
\hline Yes & $33.3(27.7-39.7)$ & $15.9(14.6-17.4)$ & $2.09(1.71-2.56)$ & $1.03(0.80-1.31)$ \\
\hline \multicolumn{5}{|c|}{ Vascular surgery performed } \\
\hline No & $27.2(25.9-28.4)$ & $16.4(16.0-16.7)$ & $1.66(1.58-1.75)$ & $1.04(0.99-1.10)$ \\
\hline Yes & $16.8(12.0-23.1)$ & $10.4(9.3-11.6)$ & $1.61(1.14-2.28)$ & $0.64(0.31-1.33)$ \\
\hline
\end{tabular}


Table 3 Mortality at 30 days and 365 days following admission in 57,110 adult ICU patients with and without atrial fibrillation (Continued)

\begin{tabular}{|c|c|c|c|c|}
\hline \multicolumn{5}{|l|}{ 365-day mortality } \\
\hline Overall & $40.9(40.0-42.3)$ & $25.4(25.0-25.8)$ & $1.61(1.55-1.67)$ & $1.03(1.00-1.07)$ \\
\hline \multicolumn{5}{|l|}{ Age } \\
\hline $15-55 \mathrm{yr}$ & $19.9(15.5-25.3)$ & $9.8(9.4-10.3)$ & $2.02(1.57-2.59)$ & $1.34(1.06-1.69)$ \\
\hline $55-71 \mathrm{yr}$ & $28.0(25.8-30.3)$ & $24.8(24.2-25.5)$ & $1.13(1.04-1.23)$ & $1.07(0.98-1.16)$ \\
\hline $71-105 \mathrm{yr}$ & $48.6(46.9-50.4)$ & $43.1(42.4-43.9)$ & $1.13(1.08-1.17)$ & $1.03(1.00-1.07)$ \\
\hline \multicolumn{5}{|l|}{ Sex } \\
\hline Female & $45.6(43.4-48.9)$ & $25.9(25.4-26.5)$ & $1.76(1.67-1.86)$ & $1.07(1.01-1.13)$ \\
\hline Male & $38.1(36.4-39.8)$ & $25.0(24.5-25.5)$ & $1.53(1.45-1.60)$ & $1.01(0.96-1.05)$ \\
\hline \multicolumn{5}{|c|}{ Prior myocardial infarction } \\
\hline Not present & $39.9(38.4-41.5)$ & $24.8(24.4-25.2)$ & $1.61(1.55-1.68)$ & $1.02(0.98-1.07)$ \\
\hline Present & $44.7(41.8-47.8)$ & $31.2(29.9-32.5)$ & $1.43(1.33-1.55)$ & $1.07(0.99-1.16)$ \\
\hline \multicolumn{5}{|c|}{ Congestive heart failure } \\
\hline Not present & $37.1(35.5-38.8)$ & $24.2(23.8-24.6)$ & $1.53(1.46-1.61)$ & $1.04(0.99-1.08)$ \\
\hline Present & $48.4(46.0--50.7)$ & $43.8(42.1-45.6)$ & $1.10(1.04-1.18)$ & $1.04(0.98-1.10)$ \\
\hline \multicolumn{5}{|c|}{ Mechanical ventilation } \\
\hline No & $38.8(38.1-41.6)$ & $21.1(20.6-21.5)$ & $1.89(1.80-1.99)$ & $1.00(0.95-1.05)$ \\
\hline Yes & $42.4(40.4-44.6)$ & $33.3(32.6-34.0)$ & $1.27(1.21-1.34)$ & $1.09(1.04-1.15)$ \\
\hline \multicolumn{5}{|c|}{ Renal replacement therapy } \\
\hline No & $39.6(38.3-41.0)$ & $24.4(24.1-24.8)$ & $1.62(1.56-1.68)$ & $1.03(0.99-1.06)$ \\
\hline Yes & $63.4(59.5-71.2)$ & $59.2(56.6-61.7)$ & $1.10(1.00-1.22)$ & $1.04(0.94-1.15)$ \\
\hline \multicolumn{5}{|c|}{ Treatment with inotropes or vasopressors } \\
\hline No & $46.6(39.2-54.6)$ & $28.7(26.4-31.2)$ & $1.62(1.35-1.95)$ & $1.00(0.82-1.21)$ \\
\hline Yes & $52.3(45.1-60.0)$ & $42.9(40.3-45.6)$ & $1.22(1.04-1.43)$ & $0.96(0.82-1.12)$ \\
\hline \multicolumn{5}{|c|}{ Non-invasive ventilation } \\
\hline No & $47.6(41.6-54.0)$ & $32.2(30.3-34.3)$ & $1.48(1.28-1.71)$ & $0.99(0.85-1.15)$ \\
\hline Yes & $55.3(45.1-66.0)$ & $48.6(44.6-52.7)$ & $1.14(0.92-1.40)$ & $0.96(0.78-1.18)$ \\
\hline \multicolumn{5}{|c|}{ Surgery performed, any type } \\
\hline No & $50.1(47.9-52.3)$ & $30.6(30.0-31.3)$ & $1.64(1.56-1.72)$ & $0.96(0.92-1.01)$ \\
\hline Yes & $34.8(33.1-36.5)$ & $22.2(21.7-22.6)$ & $1.57(1.49-1.66)$ & $1.07(1.02-1.13)$ \\
\hline \multicolumn{5}{|c|}{ Cardiac surgery performed } \\
\hline No & $47.6(46.1-49.2)$ & $27.6(27.2-28.1)$ & $1.72(1.66-1.79)$ & $1.00(0.97-1.04)$ \\
\hline Yes & $14.9(12.9-17.3)$ & $10.2(9.5-10.9)$ & $1.47(1.25-1.73)$ & $1.11(0.88-1.40)$ \\
\hline \multicolumn{5}{|c|}{ Abdominal surgery performed } \\
\hline No & $39.7(38.3-41.2)$ & $24.7(24.3-25.1)$ & $1.61(1.55-1.68)$ & $1.03(0.99-1.07)$ \\
\hline Yes & $46.4(43.2-49.7)$ & $28.6(27.7-29.5)$ & $1.62(1.50-1.75)$ & $1.04(0.97-1.12)$ \\
\hline \multicolumn{5}{|c|}{ Orthopaedic surgery performed } \\
\hline No & $40.3(38.9-41.7)$ & $25.5(25.1-25.9)$ & $1.58(1.52-1.64)$ & $1.03(0.99-1.07)$ \\
\hline Yes & $54.4(48.2-60.9)$ & $24.1(22.5-25.8)$ & $2.27(1.98-2.59)$ & $1.11(0.97-1.27)$ \\
\hline \multicolumn{5}{|c|}{ Vascular surgery performed } \\
\hline No & $41.2(39.8-42.6)$ & $25.8(25.4-26.2)$ & $1.60(1.54-1.66)$ & $1.02(0.99-1.06)$ \\
\hline Yes & $33.0(26.6-40.4)$ & 18.0 (16.6-19.5) & $1.83(1.47-2.29)$ & $1.18(0.88-1.57)$ \\
\hline
\end{tabular}

$A F$ atrial fibrillation, $R R$ relative risk

$95 \%$ confidence intervals are given in parentheses

${ }^{a}$ Reference group was non-AF patients within each stratum

${ }^{\mathrm{b}}$ Adjusted for age and sex, comorbidities, and consultations and services provided by the general practitioner 
Table 4 Mortality at 30 days and 365 days following admission in 57,110 adult ICU patients with and without pre-admission use of different drugs, according to atrial fibrillation status

\begin{tabular}{|c|c|c|c|c|}
\hline & Users, \% & Non-users, \% & Crude RR $^{a}$ & Adjusted RR $R^{a, b}$ \\
\hline \multicolumn{5}{|c|}{ 30-day mortality } \\
\hline \multicolumn{5}{|l|}{ Statins } \\
\hline No AF & $15.8(15.1-16.5)$ & $16.1(15.8-16.5)$ & $0.98(0.93-1.03)$ & $0.83(0.79-0.88)$ \\
\hline $\mathrm{AF}$ & $23.6(21.7-25.7)$ & $28.4(26.9-30.0)$ & $0.83(0.75-0.92)$ & $0.93(0.84-1.04)$ \\
\hline \multicolumn{5}{|l|}{ Aspirin } \\
\hline No AF & $20.1(19.3-20.8)$ & $15.0(14.7-15.4)$ & $1.34(1.28-1.40)$ & $0.93(0.88-0.97)$ \\
\hline AF & $28.2(26.3-30.3)$ & $25.9(24.4-27.5)$ & $1.09(0.99-1.20)$ & $0.99(0.91-1.09)$ \\
\hline \multicolumn{5}{|c|}{ Vitamin $\mathrm{K}$ antagonists } \\
\hline No AF & $23.3(21.1-25.7)$ & $15.9(15.5-1.62)$ & $1.47(1.33-1.62)$ & $1.07(0.97-1.19)$ \\
\hline$A F$ & $22.9(21.3-24.6)$ & $30.6(28.9-32.4)$ & $0.75(0.68-0.82)$ & $0.91(0.82-1.00)$ \\
\hline \multicolumn{5}{|l|}{ Beta-blockers } \\
\hline No AF & $17.2(16.4-17.9)$ & $15.8(15.4-16.1)$ & $1.09(1.02-1.12)$ & $0.91(0.86-0.95)$ \\
\hline AF & $26.4(24.8-28.1)$ & $27.3(25.5-29.2)$ & $0.97(0.88-1.06)$ & $1.07(0.98-1.18)$ \\
\hline \multicolumn{5}{|c|}{ Non-dihydropyridine CCBs } \\
\hline No AF & $21.4(18.9-24.3)$ & $16.0(15.6-16.3)$ & $1.34(1.18-1.52)$ & $1.04(0.92-1.17)$ \\
\hline AF & $24.2(20.1-28.9)$ & $27.0(25.8-28.3)$ & $0.90(0.74-1.08)$ & $0.86(0.71-1.03)$ \\
\hline \multicolumn{5}{|l|}{ Digoxin } \\
\hline No AF & $32.7(29.6-36.2)$ & $15.8(15.5-16.1)$ & $2.07(1.87-2.30)$ & $1.15(1.03-1.28)$ \\
\hline AF & $29.5(27.4-31.8)$ & $25.5(24.0-27.0)$ & $1.16(1.05-1.27)$ & $0.97(0.88-1.07)$ \\
\hline \multicolumn{5}{|l|}{ Amiodarone } \\
\hline No AF & $25.2(18.3-34.2)$ & $16.0(15.7-16.3)$ & $1.58(1.15-2.16)$ & $1.08(0.83-1.42)$ \\
\hline AF & $27.3(23.0-32.2)$ & $26.8(25.5-28.0)$ & $1.02(0.86-1.21)$ & $1.15(0.98-1.34)$ \\
\hline \multicolumn{5}{|c|}{ 365-day mortality } \\
\hline \multicolumn{5}{|l|}{ Statins } \\
\hline No AF & $24.8(24.0-25.6)$ & $25.6(25.1-26.0)$ & $0.97(0.94-1.01)$ & $0.82(0.79-0.86)$ \\
\hline AF & $36.7(34.5-39.1)$ & $43.1(41.4-44.8)$ & $0.85(0.79-0.92)$ & $0.95(0.88-1.02)$ \\
\hline \multicolumn{5}{|l|}{ Aspirin } \\
\hline No AF & $31.0(30.2-31.9)$ & $23.9(23.5-24.4)$ & $1.30(1.25-1.34)$ & $0.93(0.90-0.96)$ \\
\hline $\mathrm{AF}$ & $42.7(40.6-44.9)$ & $39.8(38.1-41.5)$ & $1.07(1.00-1.15)$ & $1.00(0.94-1.06)$ \\
\hline \multicolumn{5}{|c|}{ Vitamin $\mathrm{K}$ antagonists } \\
\hline No AF & $37.8(35.3-40.5)$ & $25.1(24.7-25.5)$ & $1.51(1.41-1.62)$ & $1.11(1.03-1.19)$ \\
\hline $\mathrm{AF}$ & $35.4(33.5-37.3)$ & $46.3(44.4-48.3)$ & $0.76(0.71-0.82)$ & $0.91(0.85-0.97)$ \\
\hline \multicolumn{5}{|l|}{ Beta-blockers } \\
\hline No AF & $26.9(26.0-27.7)$ & $25.1(24.6-25.5)$ & $1.07(1.03-1.11)$ & $0.89(0.86-0.93)$ \\
\hline $\mathrm{AF}$ & $39.4(37.6-41.3)$ & $42.7(40.7-44.8)$ & $0.92(0.86-0.99)$ & $1.00(0.95-1.07)$ \\
\hline \multicolumn{5}{|c|}{ Non-dihydropyridine CCBs } \\
\hline No AF & $33.3(30.3-36.5)$ & $25.3(24.8-25.6)$ & $1.32(1.20-1.45)$ & $1.02(0.94-1.11)$ \\
\hline AF & $39.4(34.6-44.7)$ & $41.0(39.6-42.4)$ & $0.96(0.84-1.10)$ & $0.93(0.82-1.05)$ \\
\hline \multicolumn{5}{|l|}{ Digoxin } \\
\hline No AF & $50.1(46.6-53.6)$ & $25.0(24.7-25.4)$ & $2.00(1.86-2.15)$ & $1.16(1.08-1.24)$ \\
\hline AF & $45.0(42.6-47.4)$ & $38.9(37.3-40.6)$ & $1.15(1.08-1.24)$ & $1.01(0.95-1.08)$ \\
\hline
\end{tabular}


Table 4 Mortality at 30 days and 365 days following admission in 57,110 adult ICU patients with and without pre-admission use of different drugs, according to atrial fibrillation status (Continued)

\begin{tabular}{lllll}
\hline Amiodarone & & & & \\
No AF & $44.4(35.8-53.9)$ & $25.4(25.0-25.7)$ & $1.75(1.42-2.15)$ & $1.18(0.99-1.41)$ \\
AF & $42.2(37.3-47.4)$ & $40.8(39.4-42.2)$ & $1.03(0.91-1.17)$ & $1.12(1.01-1.25)$
\end{tabular}

AF atrial fibrillation, $R R$ relative risk, CCBs calcium channel blockers

95\% confidence intervals are given in parentheses

${ }^{a}$ Reference group was non-users within each stratum

${ }^{\mathrm{b}}$ Adjusted for age and sex, comorbidities, and consultations and services by the general practitioner

note, AF per se appeared to increase mortality in younger ICU patients and in those treated with mechanical ventilation. In patients with pre-existing $A F$, preadmission users of vitamin $\mathrm{K}$ antagonists seemed to have a lower risk of ATE and had reduced mortality.

Biological mechanisms behind these findings cannot be clarified by this observational study. AF is a wellestablished risk factor for development of thromboembolic events [7]. In the general population, the stroke risk associated with $\mathrm{AF}$ is dependent on coexisting diseases, as expressed by the $\mathrm{CHA}_{2} \mathrm{DS}_{2}$-VASc score $[13,23]$. In our cohort, AF was associated with a modestly increased risk of ATE even after we adjusted for the risk factors included in the $\mathrm{CHA}_{2} \mathrm{DS}_{2}$-VASc score, indicating that patients with pre-existing $\mathrm{AF}$ are more vulnerable to the mechanisms leading to increased risk of ATE in critical illness than are nonAF patients. These mechanisms may include increased procoagulant activity induced by the inflammatory response [33]. Frailty following critical illness could also be an explanation of the increased long-term risk of ATE. Even though our results did not unambiguously demonstrate reduced risk of ATE with pre-admission use of vitamin $\mathrm{K}$ antagonists, patients with AF who were vitamin $\mathrm{K}$ antagonist users had lower mortality at both 30 days and 1 year following ICU admission, illustrating that protection from hypercoagulation could be beneficial for AF patients admitted to the ICU.

In two previous studies, researchers have reported inICU mortality ranging from $25 \%$ to $27 \%$ in patients with pre-existing AF, which is compatible with our 30 -day estimate [34, 35]. The intent of the previous studies was not to describe the prognostic impact of pre-existing AF in ICU patients, however, and no RR estimates were reported. Our finding of no excess mortality with pre-existing AF contrasts with reports of increased mortality in ICU patients with new-onset AF. Perhaps new-onset AF during ICU admission reflects greater disease severity rather than being a cause of increased mortality per se. Alternatively, patients with pre-existing AF may have adapted to the condition-physiologically or by use of medications-and could therefore be less susceptible to complications than ICU patients with new-onset AF. In ICU patients younger than
55 years of age and in those treated with mechanical ventilation, we did find increased mortality with AF. Young ICU patients have little comorbidity, and their absolute mortality is comparably low. Thus, on a relative scale, presence versus absence of AF is likely to impact mortality more in this age group than in older, multimorbid ICU patients, in whom the effect of AF is often conjugated with effects of many other conditions. The 1.12-fold relative mortality increase with AF in patients treated with mechanical ventilation may be of clinical relevance, given the high absolute mortality risk in this patient group. Tachycardia and irregular heart rate may reduce cardiac filling in patients with AF, which could be further compromised by the positive intrathoracic pressure stemming from mechanical ventilation. Alternatively, mechanical ventilation may be mainly a marker of greater disease severity during which pre-existing AF could have an impact on mortality in patients with severe haemodynamic instability due to diminished cardiac performance caused by AF itself.

Pre-admission use of beta-blockers and statins has previously been associated with favourable prognosis in a broad population of ICU patients [22, 36]. We did not find a beneficial effect of either statins or beta-blockers in patients with AF, however. Of note, our estimates in non-AF patients were comparable with those previously reported [22, 36].

\section{Strengths and limitations}

Major strengths of this study include its populationbased design, that it was conducted within a uniform health care system, the use of prospectively and independently sampled data, and virtually complete followup, thereby reducing the risk of selection problems seen in studies based on selected clinics or patient groups. AF is reliably coded in the DNPR with a positive predictive value in the range 93-99 \% [19, 37, 38].

The positive predictive value for ischaemic stroke is $88-100 \%$. Of patients coded with unspecified stroke, $57-70 \%$ have ischaemic stroke [39, 40]. As previously noted, we were unable to assess exact temporal relationships between diseases diagnosed during the same admission, and some cases of ATE may have been present at ICU admission, thereby overestimating the risk of ATE 
from AF following ICU admission. We have addressed this issue in our sensitivity analysis, but the data are likely to underestimate the true risk of ATE as we considered only ATE diagnosed after discharge from the entire hospital admission during which the patient was treated in the ICU.

The use of filled prescriptions as a proxy for use of pharmaceuticals implies that any non-adherence or discontinuation of a given drug before admission would introduce a bias towards the null. Likewise, as we did not have data on in-hospital treatments, discontinuation of a drug during admission could also lead to a downward bias. Indeed, as ICU patients often receive nutrition via a feeding tube, it is likely that many patients in this cohort have had their pre-admission oral medications paused or altered. Also, use of anticoagulants, such as low molecular weight heparins, during the ICU admission could influence the risks of ATE and death in patients with AF, but we lacked data to elaboration on this potential mechanism. Users of prophylactic medications may in general have greater awareness of own health and disease than non-users. The structure of the Danish health care system with tax-funded medical care and partial reimbursement of prescription drugs probably outbalances these effects to some extent, and previous research has shown that users of medications such as statins in Denmark in fact have an unhealthier lifestyle than non-users [41]. Still, we cannot rule out that our finding of a beneficial effect of pre-admission use of vitamin $\mathrm{K}$ antagonists is affected by a 'healthy user/ adherer bias', or rather a 'frail non-user bias'.

The detailed registries and our large dataset allowed us to control extensively for potential confounders, of which the disease categories included in the Charlson comorbidity index have been validated with a mean positive predictive value of $98 \%$ [42]. Because there is a substantial overlap in the disease categories included in the Charlson comorbidity index and the $\mathrm{CHA}_{2} \mathrm{DS}_{2}$-VASc score, we also expect the coding of the latter to have a high validity, albeit residual confounding and confounding from unmeasured variables, such as diseases managed solely by the patient's GP (which includes, e.g., uncomplicated hypertension and uncomplicated type 2 diabetes) and lifestyle factors, could still influence our estimates.

\section{Conclusions}

Pre-existing AF is common in ICU patients and predicts increased risk of ATE and death. However, coexisting diseases and advanced age in patients with AF explain much of these findings. AF was an independent risk factor for ATE at 1 year following ICU admission and for death at 30 days and 1 year following admission in ICU patients treated with mechanical ventilation and in patients younger than 55 years of age. Pre-admission use of vitamin $\mathrm{K}$ antagonists improved the prognosis for patients with AF. At present, there are no standards for management of pre-existing AF in ICU patients, and this study highlights the urgent need for evidence-based clinical guidelines in this area.

\section{Key messages}

- This population-based study shows that pre-existing $\mathrm{AF}$ is common in ICU patients and a marker of increased risk of ATE and death.

- The increased risk of ATE and death is largely explained by older age and more comorbidities in patients with AF.

- Pre-existing AF per se predicts increased mortality in ICU patients younger than 55 years of age and in ICU patients treated with mechanical ventilation.

- Pre-admission use of vitamin K antagonists improves the prognosis for ICU patients with pre-existing AF.

\section{Additional file}

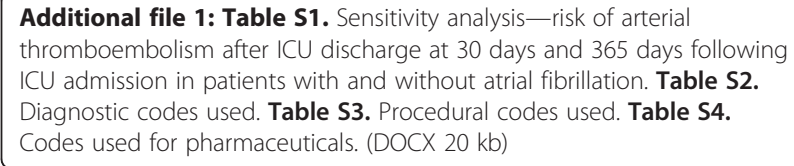

\section{Abbreviations}

AF: Atrial fibrillation; ATE: Arterial thromboembolism; CCB: Calcium channel blocker; $\mathrm{CHA}_{2} \mathrm{DS}_{2}$-VASc: Congestive heart failure, hypertension, age, diabetes, prior stroke/transient ischaemic attack/thromboembolism, vascular disease, and sex category; Cl: Confidence interval; CRR: Cumulative risk ratio; DID: Danish Intensive Care Database; DNPR: Danish National Patient Register; GP: General practitioner; ICU: Intensive care unit; IQR: Interquartile range; RR: Relative risk.

\section{Competing interests}

The authors declare that they have no competing interests.

\section{Authors' contributions}

$J G$ participated in conceptualizing and designing the study, performed the statistical analyses, interpreted the data, and wrote the first draft of the manuscript. CFC participated in conceptualizing and designing the study, helped in interpreting the data, and participated in critically reviewing and writing the manuscript. BSR participated in conceptualizing and designing the study, helped in interpreting the data, and participated in critically reviewing and writing the manuscript. LHR participated in conceptualizing and designing the study, helped in interpreting the data, provided funding, and participated in critically reviewing and writing the manuscript. RWT participated in conceptualizing and designing the study, helped in interpreting the data, provided funding, participated in critically reviewing and writing the manuscript, and supervised the study. All authors read and approved the final manuscript.

\section{Acknowledgements}

The study was supported by research grants from the Danish Council for Strategic Research (grant 09-066965), the Clinical Epidemiological Research Foundation, the Karen Elise Jensen Foundation, and the Heinrich Kopp Foundation. The sponsors did not have a role in any phase of the study. We are thankful to Thomas B. Rasmussen, MSc, for assistance with data extraction. 


\section{Author details}

'Department of Clinical Epidemiology, Institute of Clinical Medicine, Aarhus University Hospital, Olof Palmes Allé 43-45, DK-8200 Aarhus N, Denmark. ${ }^{2}$ Department of Cardiology, Aalborg University Hospital, Hobrovej 18-22, DK-9000 Aalborg, Denmark. ${ }^{3}$ Department of Anaesthesia and Intensive Care Medicine, Aalborg University Hospital, Hobrovej 18-22, DK-9000 Aalborg, Denmark. ${ }^{4}$ Aalborg Atrial Fibrillation Study Group, Aalborg University Hospital Science and Innovation Centre, Søndre Skovvej 15, DK-9000 Aalborg, Denmark. ${ }^{5}$ Faculty of Medicine, Aalborg University, Niels Jernes Vej 10, DK-9220 Aalborg Øst, Denmark.

Received: 5 May 2015 Accepted: 21 July 2015

\section{Published online: 19 August 2015}

\section{References}

1. Christiansen CF, Christensen S, Johansen MB, Larsen KM, Tønnesen E, Sørensen HT. The impact of pre-admission morbidity level on 3-year mortality after intensive care: a Danish cohort study. Acta Anaesthesiol Scand. 2011;55:962-70.

2. Ho KM, Knuiman M, Finn J, Webb SA. Estimating long-term survival of critically ill patients: the PREDICT model. PLoS One. 2008;3:e3226.

3. Flaatten $\mathrm{H}$. Intensive care in the very old: are we prepared? Acta Anaesthesiol Scand. 2007:51:519-21

4. Go AS, Hylek EM, Phillips KA, Chang Y, Henault LE, Selby JV, et al. Prevalence of diagnosed atrial fibrillation in adults: national implications for rhythm management and stroke prevention. The AnTicoagulation and Risk Factors in Atrial Fibrillation (ATRIA) Study. JAMA. 2001;285:2370-5.

5. Krijthe BP, Kunst A, Benjamin EJ, Lip GYH, Franco OH, Hofman A, et al. Projections on the number of individuals with atrial fibrillation in the European Union, from 2000 to 2060. Eur Heart J. 2013:34:2746-51.

6. Benjamin EJ, Wolf PA, D'Agostino RB, Silbershatz H, Kannel WB, Levy D. Impact of atrial fibrillation on the risk of death: the Framingham heart study. Circulation. 1998;98:946-52

7. Wolf PA, Abbott RD, Kannel WB. Atrial fibrillation as an independent risk factor for stroke: the Framingham Study. Stroke. 1991;22:983-8.

8. Wang TJ, Larson MG, Levy D, Vasan RS, Leip EP, Wolf PA, et al. Temporal relations of atrial fibrillation and congestive heart failure and their joint influence on mortality: the Framingham Heart Study. Circulation. 2003;107:2920-5.

9. Meierhenrich R, Steinhilber E, Eggermann C, Weiss M, Voglic S, Bögelein D, et al. Incidence and prognostic impact of new-onset atrial fibrillation in patients with septic shock: a prospective observational study. Crit Care. 2010;14:R108.

10. Seguin $P$, Signouret $T$, Laviolle B, Branger B, Malledant $Y$. Incidence and risk factors of atrial fibrillation in a surgical intensive care unit. Crit Care Med. 2004;32:722-6.

11. Esmon CT. The impact of the inflammatory response on coagulation. Thromb Res. 2004;114:321-7.

12. Jahangir A, Lee V, Friedman PA, Trusty JM, Hodge DO, Kopecky SL, et al. Long-term progression and outcomes with aging in patients with lone atrial fibrillation: a 30-year follow-up study. Circulation. 2007;115:3050-6.

13. Lip GYH, Nieuwlaat R, Pisters R, Lane DA, Crijns HJGM. Refining clinical risk stratification for predicting stroke and thromboembolism in atrial fibrillation using a novel risk factor-based approach: the euro heart survey on atrial fibrillation. Chest. 2010;137:263-72.

14. Stefansdottir H, Aspelund T, Gudnason V, Arnar DO. Trends in the incidence and prevalence of atrial fibrillation in Iceland and future projections. Europace. 2011;13:1110-7.

15. Nielsson MS, Christiansen CF, Johansen MB, Rasmussen BS, Tønnesen E, Nørgaard M. Mortality in elderly ICU patients: a cohort study. Acta Anaesthesiol Scand. 2014;58:19-26.

16. Rønholm E, Christensen S, Andersen JS, Jensen RH, Sørensen HT. Clinical database for intensive care patients. Ugeskr Laeger. 2007;169:724-7. Danish.

17. Lynge E, Sandegaard JL, Rebolj M. The Danish National Patient Register. Scand J Public Health. 2011;39:30-3.

18. Frost $L$, Hune $L$, Vestergaard P. Overweight and obesity as risk factors for atrial fibrillation or flutter: the Danish Diet, Cancer, and Health Study. Am J Med. 2005;118:489-95.

19. Rix TA, Riahi S, Overvad K, Lundbye-Christensen S, Schmidt EB, Joensen AM. Validity of the diagnoses atrial fibrillation and atrial flutter in a Danish patient registry. Scand Cardiovasc J. 2012;46:149-53.
20. Johannesdottir SA, Horváth-Puhó E, Ehrenstein V, Schmidt M, Pedersen L, Sørensen HT. Existing data sources for clinical epidemiology: The Danish National database of reimbursed prescriptions. Clin Epidemiol. 2012;4:303-13.

21. Hansen ML, Gadsbøll N, Gislason GH, Abildstrom SZ, Schramm TK, Folke F, et al. Atrial fibrillation pharmacotherapy after hospital discharge between 1995 and 2004: a shift towards beta-blockers. Europace. 2008;10:395-402.

22. Christensen S, Thomsen RW, Johansen MB, Pedersen L, Jensen R, Larsen KM, et al. Preadmission statin use and one-year mortality among patients in intensive care - a cohort study. Crit Care. 2010;14:R29.

23. Olesen JB, Lip GYH, Hansen ML, Hansen PR, Tolstrup JS, Lindhardsen J, et al. Validation of risk stratification schemes for predicting stroke and thromboembolism in patients with atrial fibrillation: nationwide cohort study. BMJ. 2011;342:d124.

24. Charlson ME, Pompei P, Ales KL, MacKenzie CR. A new method of classifying prognostic comorbidity in longitudinal studies: development and validation. J Chronic Dis. 1987;40:373-83.

25. De Groot V, Beckerman H, Lankhorst GJ, Bouter LM. How to measure comorbidity: a critical review of available methods. J Clin Epidemiol. 2003:56:221-9.

26. Christensen $\mathrm{S}$, Johansen MB, Christiansen CF, Jensen R, Lemeshow S Comparison of Charlson comorbidity index with SAPS and APACHE scores for prediction of mortality following intensive care. Clin Epidemiol. 2011;3:203-11.

27. Andersen JS, Olivarius NF, Krasnik A. The Danish National Health Service Register. Scand J Public Health. 2011;39:34-7.

28. Pedersen CB, Gøtzsche H, Møller JO, Mortensen PB. The Danish Civil Registration System: a cohort of eight million persons. Dan Med Bull. 2006;53:441-9.

29. Andersen PK, Geskus RB, de Witte T, Putter H. Competing risks in epidemiology: possibilities and pitfalls. Int J Epidemiol. 2012;41:861-70

30. Parner ET, Andersen PK. Regression analysis of censored data using pseudo-observations. Stata J. 2010;10:408-22.

31. Klein JP, Andersen PK. Regression modeling of competing risks data based on pseudovalues of the cumulative incidence function. Biometrics. 2005;61:223-9.

32. Klein JP, Logan B, Harhoff $M$, Andersen PK. Analyzing survival curves at a fixed point in time. Stat Med. 2007;26:4505-19.

33. Amaral A, Opal SM, Vincent JL. Coagulation in sepsis. Intensive Care Med. 2004;30:1032-40.

34. Kanji S, Williamson DR, Yaghchi BM, Albert M, Mclntyre L. Epidemiology and management of atrial fibrillation in medical and noncardiac surgical adult intensive care unit patients. J Crit Care. 2012;27:326. e1-e8.

35. Goodman S, Shirov T, Weissman C. Supraventricular arrhythmias in intensive care unit patients: short and long-term consequences. Anesth Analg. 2007;104:880-6.

36. Christensen S, Johansen MB, Tønnesen E, Larsson A, Pedersen L, Lemeshow $\mathrm{S}$, et al. Preadmission beta-blocker use and 30-day mortality among patients in intensive care: a cohort study. Crit Care. 2011;15:R87.

37. Frost $L$, Vestergaard P. Alcohol and risk of atrial fibrillation or flutter: a cohort study. Arch Intern Med. 2004;164:1993-8.

38. Frost L, Andersen LV, Vestergaard P, Husted S, Mortensen LS. Trend in mortality after stroke with atrial fibrillation. Am J Med. 2007;120:47-53.

39. Johnsen SP, Overvad K, Sørensen HT, Tjønneland A, Husted SE. Predictive value of stroke and transient ischemic attack discharge diagnoses in the Danish National Registry of Patients. J Clin Epidemiol. 2002;55:602-7.

40. Krarup LH, Boysen G, Janjua H, Prescott E, Truelsen T. Validity of stroke diagnoses in a national register of patients. Neuroepidemiology. 2007;28:150-4.

41. Thomsen RW, Nielsen RB, Nørgaard M, Horsdal HT, Stürmer T, Larsen FB, et al. Lifestyle profile among statin users. Epidemiology. 2013;24:619-20.

42. Thygesen SK, Christiansen CF, Christensen S, Lash TL, Sørensen HT. The predictive value of ICD-10 diagnostic coding used to assess Charlson comorbidity index conditions in the population-based Danish National Registry of Patients. BMC Med Res Methodol. 2011;11:83. 